\title{
Heavy quarkonium photoproduction in ultrarelativistic heavy ion collisions
}

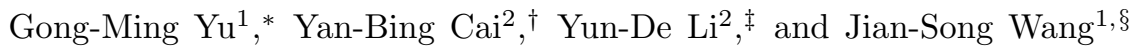 \\ ${ }^{1}$ Institute of Modern Physics, Chinese Academy of Sciences, Lanzhou 730000, China \\ ${ }^{2}$ Department of Physics, Yunnan University, Kunming 650091, China
}

\begin{abstract}
Based on the factorization formalism of non-relativistic Quantum Chromodynamics (NRQCD), we calculate the production cross section for the charmonium $\left(J / \psi, \psi(2 S), \chi_{c J}, \eta_{c}\right.$, and $\left.h_{c}\right)$ and the bottomonium $\left(\Upsilon(n S), \chi_{b J}, \eta_{b}\right.$, and $\left.h_{b}\right)$ produced by the hard photoproduction processes and fragmentation processes in relativistic heavy ion collisions. It is shown that the existing experimental data on heavy quarkonium production at Large Hadron Collider (LHC) can be described in the framework of the NRQCD formalism, and the phenomenological value of matrix element for color-singlet and color-octet components give main contribution. The numerical results of photoproduction processes and fragmentation processes for the heavy quarkonium production become prominent in $\mathrm{p}-\mathrm{p}$ collisions and $\mathrm{Pb}-\mathrm{Pb}$ collisions at Large Hadron Collider (LHC) energies.
\end{abstract}

\section{INTRODUCTION}

Hadronic processes for large transverse momentum heavy quarkonium is a vital goal in ultrarelativistic heavy ion collisions at Large Hadron Collider (LHC). In the last years, the measurements reported by the ALICE collaboration 1 5], CMS collaboration [6 9], ATLAS collaboration 10, 11], and LHCb collaboration 1214 at the Large Hadron Collider (LHC) have posed significant challenges to our understanding of the heavy quarkonium production. Furthermore, several theoretical approaches have been proposed for the calculation of these states, as for instance, the color-singlet mechanism [15, 16], the color-octet mechanism [17 22], the color evaporation mechanism 23, 24], the colordipole mechanism 25 28], the mixed heavy-quark hybrids mechanism 29], the recombination mechanism 30 32], the photoproduction mechanism [33 49], the potential non-relativistic Quantum Chromodynamics (pNRQCD) approach 50 52], the transverse-momentum-dependent factorization approach [53], the transport approach 54[56], the $k_{T}$-factorization approach 57 61], the fragmentation approach [62 74], and the non-relativistic Quantum Chromodynamics (NRQCD) approach 75 95]. Among them, the non-relativistic Quantum Chromodynamics (NRQCD) approach is the most successful in phenomenological study. According to the non-relativistic Quantum Chromodynamics (NRQCD) in addition to color-singlet component one has to take into account contributions of color-octet components with the nonperturbative longdistance matrix elements. This formalism implies a separation of short-distance coefficients, which can be calculated perturbatively as expansions in the running coupling constant $\alpha_{s}$, from long-distance matrix elements, which can be extracted from experiment. The longdistance matrix elements are process-independent, and

\footnotetext{
* ygmanan@163.com

† myparticle@163.com

$\ddagger$ yndxlyd@163.com

$\S$ jswang@impcas.ac.cn
}

can be classified in terms of their scaling in $v$, the relative velocity of the heavy quarks in the bound state. A crucial feature for this formalism is that it takes into account the $Q \bar{Q}$ Fock space [77], which is spanned by the states $\left.|n\rangle=||^{2 S+1} L_{J}^{(c)}\right\rangle$ with definite spin $S$, orbital angular momentum $L$, total angular momentum $J$, and color multiplicity $c$. In particular, this formalism predicts the existence of color-octet processes by the nonperturbative emission of soft gluons in nature.

Although considerable efforts both in experiment and theory, the heavy quarkonium production mechanism is still not fully understood. In the present work, we extend the hard photoproduction mechanism 96 98] which plays a fundamental role in the electron-proton deep inelastic scattering at the Hadron Electron Ring Accelerator to the heavy quarkonium production in p-p collisions and $\mathrm{Pb}-\mathrm{Pb}$ collisions at Large Hadron Collider (LHC) energies. At high energies, the nucleus (the charged partons) can emit high-energy photons (and hadron-like photons) in ultrarelativistic nucleus-nucleus collisions. The hard photoproduction processes may be direct and resolved that are sensitive to the gluon distribution in the nucleus. In the hard direct photoproduction processes, the highenergy photon emitted from the nucleus (the charged parton of the incident nucleus) interacts with the parton of another incident nucleus. In the hard resolved photoproduction processes, the uncertainty principle allows the high-energy hadronlike photon emitted from the nucleus (the charged parton of the incident nucleus) for a short time to fluctuate into a quark-antiquark pair which then interacts with the partons of another incident nucleus. For the fragmentation processes, the jets can produced by the hard photoproduction processes and the hard resolved photoproduction processes. Subsequently the jets will fragment into heavy quarkonium.

The paper is organized as follows. In Sec.II we present the photoproduction of heavy quarkonium at Large Hadron Collider (LHC) energies. The production rate for fragmentation processes is also discussed. The numerical results for large- $p_{T}$ heavy quarkonium in p-p collisions and $\mathrm{Pb}-\mathrm{Pb}$ collisions at Large Hadron Collider 
(LHC) energies are plotted in Sec.III. Finally, the conclusion is given in Sec.IV.

\section{PHOTOPRODUCTION PROCESSES FOR LARGE- $P_{T}$ HEAVY QUARKONIUM}

The non-relativistic Quantum Chromodynamics (NRQCD) approach 99 which is introduced in 1995 has become the standard framework to study heavyquarkonium physics. In the non-relativistic Quantum Chromodynamics (NRQCD), the Fock state structure of heavy quarkonium state is 99,100 .

$$
\begin{aligned}
\left.\left.\right|^{2 S+1} L_{J}\right\rangle= & \left.\left.\mathcal{O}(1)\right|^{2 S+1} L_{J}^{[1]}\right\rangle \\
& \left.+\left.\mathcal{O}(v)\right|^{2 S+1}(L \pm 1)_{J^{\prime}}^{[8]} g\right\rangle \\
& \left.+\left.\mathcal{O}\left(v^{2}\right)\right|^{2(S \pm 1)+1}(L \pm 1)_{J^{\prime}}^{[8]} g\right\rangle \\
& \left.+\left.\mathcal{O}\left(v^{2}\right)\right|^{2 S+1} L_{J}^{[1,8]} g g\right\rangle \\
& +\cdots \cdots,
\end{aligned}
$$

where $v$ is the relative velocity of the heavy quarks in heavy quarkonium.

The wavefunctions of the charmonium $(J / \psi, \psi(2 s)$, $\chi_{c J}, \eta_{c}$, and $\left.h_{c}\right)$ can be written as

$$
\begin{aligned}
& |J / \psi\rangle=\left\langle\mathcal{O}^{J / \psi}\left[{ }^{3} S_{1}^{[1]}\right]\right\rangle\left|Q \bar{Q}\left[{ }^{3} S_{1}^{[1]}\right]\right\rangle \\
& +\left\langle\mathcal{O}^{J / \psi}\left[{ }^{1} S_{0}^{[8]}\right]\right\rangle\left|Q \bar{Q}\left[{ }^{1} S_{0}^{[8]}\right] g\right\rangle \\
& +\left\langle\mathcal{O}^{J / \psi}\left[{ }^{3} S_{1}^{[8]}\right]\right\rangle\left|Q \bar{Q}\left[{ }^{3} S_{1}^{[8]}\right] g g\right\rangle \\
& +\sum_{J^{\prime}}\left\langle\mathcal{O}^{J / \psi}\left[{ }^{3} P_{J^{\prime}}^{[8]}\right]\right\rangle\left|Q \bar{Q}\left[{ }^{3} P_{J^{\prime}}^{[8]}\right] g\right\rangle \\
& +\cdots \cdots, \\
& \left.\left.|\psi(2 S)\rangle=\left\langle\mathcal{O}^{\psi(2 S)}\left[{ }^{3} S_{1}^{[1]}\right]\right\rangle \mid Q \bar{Q}^{[3} S_{1}^{[1]}\right]\right\rangle \\
& +\left\langle\mathcal{O}^{\psi(2 S)}\left[{ }^{1} S_{0}^{[8]}\right]\right\rangle\left|Q \bar{Q}\left[{ }^{1} S_{0}^{[8]}\right] g\right\rangle \\
& +\left\langle\mathcal{O}^{\psi(2 S)}\left[{ }^{3} S_{1}^{[8]}\right]\right\rangle\left|Q \bar{Q}\left[{ }^{3} S_{1}^{[8]}\right] g g\right\rangle \\
& +\sum_{J^{\prime}}\left\langle\mathcal{O}^{\psi(2 S)}\left[{ }^{3} P_{J^{\prime}}^{[8]}\right]\right\rangle\left|Q \bar{Q}\left[{ }^{3} P_{J^{\prime}}^{[8]}\right] g\right\rangle \\
& +\cdots \cdots, \\
& \left|\chi_{c J}\right\rangle=\left\langle\mathcal{O}^{\chi_{c J}}\left[{ }^{3} P_{J}^{[1]}\right]\right\rangle\left|Q \bar{Q}\left[{ }^{3} P_{J}^{[1]}\right]\right\rangle
\end{aligned}
$$

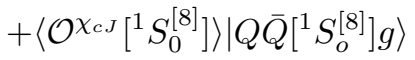

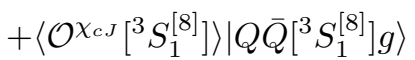

$$
\begin{aligned}
& +\left\langle\mathcal{O}^{\chi_{c J}}\left[{ }^{1} P_{1}^{[8]}\right]\right\rangle\left|Q \bar{Q}\left[{ }^{1} P_{1}^{[8]}\right] g\right\rangle
\end{aligned}
$$

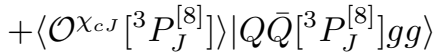

$$
\begin{aligned}
& +\left\langle\mathcal{O}^{\chi_{c J}}\left[{ }^{3} D_{J}^{[8]}\right]\right\rangle\left|Q \bar{Q}\left[{ }^{3} D_{J}^{[8]}\right] g\right\rangle \\
& +\cdots \cdots \text {, }
\end{aligned}
$$

$$
\begin{aligned}
\left|\eta_{c}\right\rangle= & \left\langle\mathcal{O}^{\eta_{c}}\left[{ }^{1} S_{0}^{[1]}\right]\right\rangle\left|Q \bar{Q}\left[{ }^{1} S_{0}^{[1]}\right]\right\rangle \\
& +\left\langle\mathcal{O}^{\eta_{c}}\left[{ }^{1} S_{0}^{[8]}\right]\right\rangle\left|Q \bar{Q}\left[{ }^{1} S_{0}^{[8]}\right] g\right\rangle \\
& +\left\langle\mathcal{O}^{\eta_{c}}\left[{ }^{3} S_{1}^{[8]}\right]\right\rangle\left|Q \bar{Q}\left[{ }^{3} S_{1}^{[8]}\right] g\right\rangle \\
& +\left\langle\mathcal{O}^{\eta_{c}}\left[{ }^{1} P_{1}^{[8]}\right]\right\rangle\left|Q \bar{Q}\left[{ }^{1} P_{1}^{[8]}\right] g\right\rangle \\
& +\cdots \cdots, \\
\left|h_{c}\right\rangle= & \left\langle\mathcal{O}^{h_{c}}\left[{ }^{1} P_{1}^{[1]}\right]\right\rangle\left|Q \bar{Q}\left[{ }^{1} P_{1}^{[1]}\right] g\right\rangle \\
& +\left\langle\mathcal{O}^{h_{c}}\left[{ }^{1} S_{0}^{[8]}\right]\right\rangle\left|Q \bar{Q}\left[{ }^{1} S_{0}^{[8]}\right] g\right\rangle \\
& +\cdots \cdots,
\end{aligned}
$$

where $\left\langle\mathcal{O}^{H}\left[{ }^{2 S+1} L_{J}^{[1,8]}\right]\right\rangle$ is the long-distance matrix element for the charmonium [18, 19, 90 94, 101, 102], and the long-distance matrix elements for the $J / \psi$ meson are given by

$$
\begin{aligned}
\left\langle\mathcal{O}^{J / \psi}\left[{ }^{3} S_{1}^{[1]}\right]\right\rangle & =1.2 \mathrm{GeV}^{3}, \\
\left\langle\mathcal{O}^{J / \psi}\left[{ }^{1} S_{0}^{[8]}\right]\right\rangle & =(0.0180 \pm 0.0087) \mathrm{GeV}^{3}, \\
\left\langle\mathcal{O}^{J / \psi}\left[{ }^{3} S_{1}^{[8]}\right]\right\rangle & =(0.0013 \pm 0.0013) \mathrm{GeV}^{3}, \\
\left\langle\mathcal{O}^{J / \psi}\left[{ }^{3} P_{0}^{[8]}\right]\right\rangle & =(0.0180 \pm 0.0087) m_{c}^{2} \cdot \mathrm{GeV}^{3}, \\
\left\langle\mathcal{O}^{J / \psi}\left[{ }^{3} P_{1}^{[8]}\right]\right\rangle & =3 \times(0.0180 \pm 0.0087) m_{c}^{2} \cdot \mathrm{GeV}^{3}, \\
\left\langle\mathcal{O}^{J / \psi}\left[{ }^{3} P_{2}^{[8]}\right]\right\rangle & =5 \times(0.0180 \pm 0.0087) m_{c}^{2} \cdot \mathrm{GeV}^{3},
\end{aligned}
$$

for $\psi(2 S)$ meson,

$$
\begin{aligned}
\left\langle\mathcal{O}^{\psi(2 S)}\left[{ }^{3} S_{1}^{[1]}\right]\right\rangle & =0.76 \mathrm{GeV}^{3}, \\
\left\langle\mathcal{O}^{\psi(2 S)}\left[{ }^{1} S_{0}^{[8]}\right]\right\rangle & =(0.0080 \pm 0.0067) \mathrm{GeV}^{3}, \\
\left\langle\mathcal{O}^{\psi(2 S)}\left[{ }^{3} S_{1}^{[8]}\right]\right\rangle & =(0.00330 \pm 0.00021) \mathrm{GeV}^{3}, \\
\left\langle\mathcal{O}^{\psi(2 S)}\left[{ }^{3} P_{0}^{[8]}\right]\right\rangle & =(0.0080 \pm 0.0067) m_{c}^{2} \cdot \mathrm{GeV}^{3}, \\
\left\langle\mathcal{O}^{\psi(2 S)}\left[{ }^{3} P_{1}^{[8]}\right]\right\rangle & =3 \times(0.0080 \pm 0.0067) m_{c}^{2} \cdot \mathrm{GeV}^{3}, \\
\left\langle\mathcal{O}^{\psi(2 S)}\left[{ }^{3} P_{2}^{[8]}\right]\right\rangle & =5 \times(0.0080 \pm 0.0067) m_{c}^{2} \cdot \mathrm{GeV}^{3},
\end{aligned}
$$

for $\chi_{c J}$ meson,

$$
\begin{aligned}
\left\langle\mathcal{O}^{\chi_{c 0}}\left[{ }^{3} P_{0}^{[1]}\right]\right\rangle & =0.054 m_{c}^{2} \cdot \mathrm{GeV}^{3}, \\
\left\langle\mathcal{O}^{\chi_{c 0}}\left[{ }^{3} S_{1}^{[8]}\right]\right\rangle & =(0.00187 \pm 0.00025) \mathrm{GeV}^{3}, \\
\left\langle\mathcal{O}^{\chi_{c 1}}\left[{ }^{3} P_{1}^{[1]}\right]\right\rangle & =3 \times 0.054 m_{c}^{2} \cdot G e V^{3}, \\
\left\langle\mathcal{O}^{\chi_{c 1}}\left[{ }^{3} S_{1}^{[8]}\right]\right\rangle & =3 \times(0.00187 \pm 0.00025) \mathrm{GeV}^{3}, \\
\left\langle\mathcal{O}^{\chi_{c 2}}\left[{ }^{3} P_{1}^{[1]}\right]\right\rangle & =5 \times 0.054 m_{c}^{2} \cdot \mathrm{GeV}^{3}, \\
\left\langle\mathcal{O}^{\chi_{c 2}}\left[{ }^{3} S_{1}^{[8]}\right]\right\rangle & =5 \times(0.00187 \pm 0.00025) \mathrm{GeV}^{3},
\end{aligned}
$$



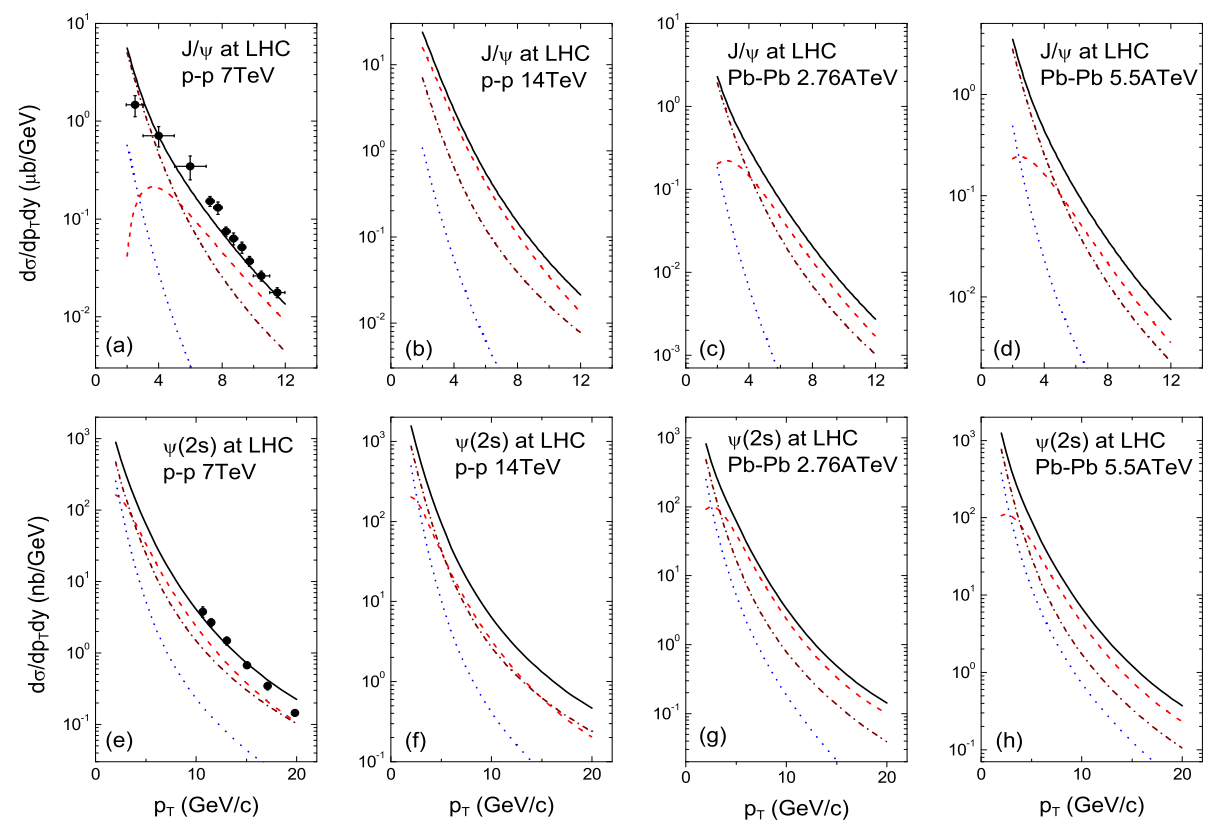

FIG. 1. The invariant cross section of large- $p_{T} J / \psi$ and $\psi(2 S)$ production for p-p collisions $(\sqrt{s}=7.0 T e V$ and $\sqrt{s}=$ 14.0TeV $)$ and $\mathrm{Pb}-\mathrm{Pb}$ collisions $(\sqrt{s}=2.76 \mathrm{TeV}$ and $\sqrt{s}=5.5 \mathrm{TeV})$ at LHC. The dashed line (red line) for the initial parton hard scattering processes (LO+LO-fra.), the dotted line (blue line) for the semielastic photoproduction processes (semi.(dir.+res.)+semi.(dir.+res.)-fra.), the dashed-dotted line (wine line) for the inelastic photoproduction processes (inel.(dir.+res.)+inel.(dir.+res.)-fra.), and the solid line (black) is for the sum of the above processes. The $J / \psi$ data points are from the ALICE Collaboration[1], and the $\psi(2 S)$ data points are from the CMS Collaboration [8].

for $\eta_{c}$ meson,

$$
\begin{aligned}
\left\langle\mathcal{O}^{\eta_{c}}\left[{ }^{1} S_{0}^{[1]}\right]\right\rangle & =\frac{1}{3} \times 1.2 \mathrm{GeV}^{3}, \\
\left\langle\mathcal{O}^{\eta_{c}}\left[{ }^{1} S_{0}^{[8]}\right]\right\rangle & =\frac{1}{3} \times(0.0013 \pm 0.0013) \mathrm{GeV}^{3}, \\
\left\langle\mathcal{O}^{\eta_{c}}\left[{ }^{3} S_{1}^{[8]}\right]\right\rangle & =(0.0180 \pm 0.0087) \mathrm{GeV}^{3}, \\
\left\langle\mathcal{O}^{\eta_{c}}\left[{ }^{1} P_{1}^{[8]}\right]\right\rangle & =3 \times(0.0180 \pm 0.0087) m_{c}^{2} \cdot \mathrm{GeV}^{3},
\end{aligned}
$$

and for $h_{c}$ meson,

$$
\begin{aligned}
& \left\langle\mathcal{O}^{h_{c}}\left[{ }^{1} P_{1}^{[1]}\right]\right\rangle=3 \times 0.54 m_{c}^{2} \cdot \mathrm{GeV}^{3}, \\
& \left\langle\mathcal{O}^{h_{c}}\left[{ }^{1} S_{0}^{[8]}\right]\right\rangle=3 \times(0.00187 \pm 0.00025) \mathrm{GeV}^{3},
\end{aligned}
$$

here $m_{c}$ is the charm quark mass.

The wavefunctions of the bottomonium $\left(\Upsilon(n s), \chi_{b J}\right.$, $\eta_{b}$, and $h_{b}$ ) can be written as

$$
\begin{aligned}
|\Upsilon(n S)\rangle= & \left\langle\mathcal{O}^{\Upsilon(n S)}\left[{ }^{3} S_{1}^{[1]}\right]\right\rangle\left|Q \bar{Q}\left[{ }^{3} S_{1}^{[1]}\right]\right\rangle \\
& +\left\langle\mathcal{O}^{\Upsilon(n S)}\left[{ }^{1} S_{0}^{[8]}\right]\right\rangle\left|Q \bar{Q}\left[{ }^{1} S_{0}^{[8]}\right] g\right\rangle \\
& +\left\langle\mathcal{O}^{\Upsilon(n S)}\left[{ }^{3} S_{1}^{[8]}\right]\right\rangle\left|Q \bar{Q}\left[{ }^{3} S_{1}^{[8]}\right] g g\right\rangle \\
& +\sum_{J^{\prime}}\left\langle\mathcal{O}^{\Upsilon(n S)}\left[{ }^{3} P_{J^{\prime}}^{[8]}\right]\right\rangle\left|Q \bar{Q}\left[{ }^{3} P_{J^{\prime}}^{[8]}\right] g\right\rangle \\
& +\cdots \cdots
\end{aligned}
$$

$$
\begin{aligned}
& \left|\chi_{b J}\right\rangle=\left\langle\mathcal{O}^{\chi_{b J}}\left[{ }^{3} P_{J}^{[1]}\right]\right\rangle\left|Q \bar{Q}\left[{ }^{3} P_{J}^{[1]}\right]\right\rangle \\
& +\left\langle\mathcal{O}^{\chi_{b J}}\left[{ }^{1} S_{0}^{[8]}\right]\right\rangle\left|Q \bar{Q}\left[{ }^{1} S_{o}^{[8]}\right] g\right\rangle
\end{aligned}
$$

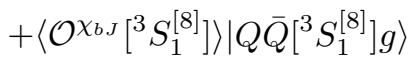

$$
\begin{aligned}
& +\left\langle\mathcal{O}^{\chi b J}\left[{ }^{1} P_{1}^{[8]}\right]\right\rangle\left|Q \bar{Q}\left[{ }^{1} P_{1}^{[8]}\right] g\right\rangle \\
& +\left\langle\mathcal{O}^{\chi \chi_{b J}}\left[{ }^{3} P_{J}^{[8]}\right]\right\rangle\left|Q \bar{Q}\left[{ }^{3} P_{J}^{[8]}\right] g g\right\rangle \\
& +\left\langle\mathcal{O}^{\chi \chi_{b J}}\left[{ }^{3} D_{J}^{[8]}\right]\right\rangle\left|Q \bar{Q}\left[{ }^{3} D_{J}^{[8]}\right] g\right\rangle \\
& +\cdots \cdot \text {, }
\end{aligned}
$$



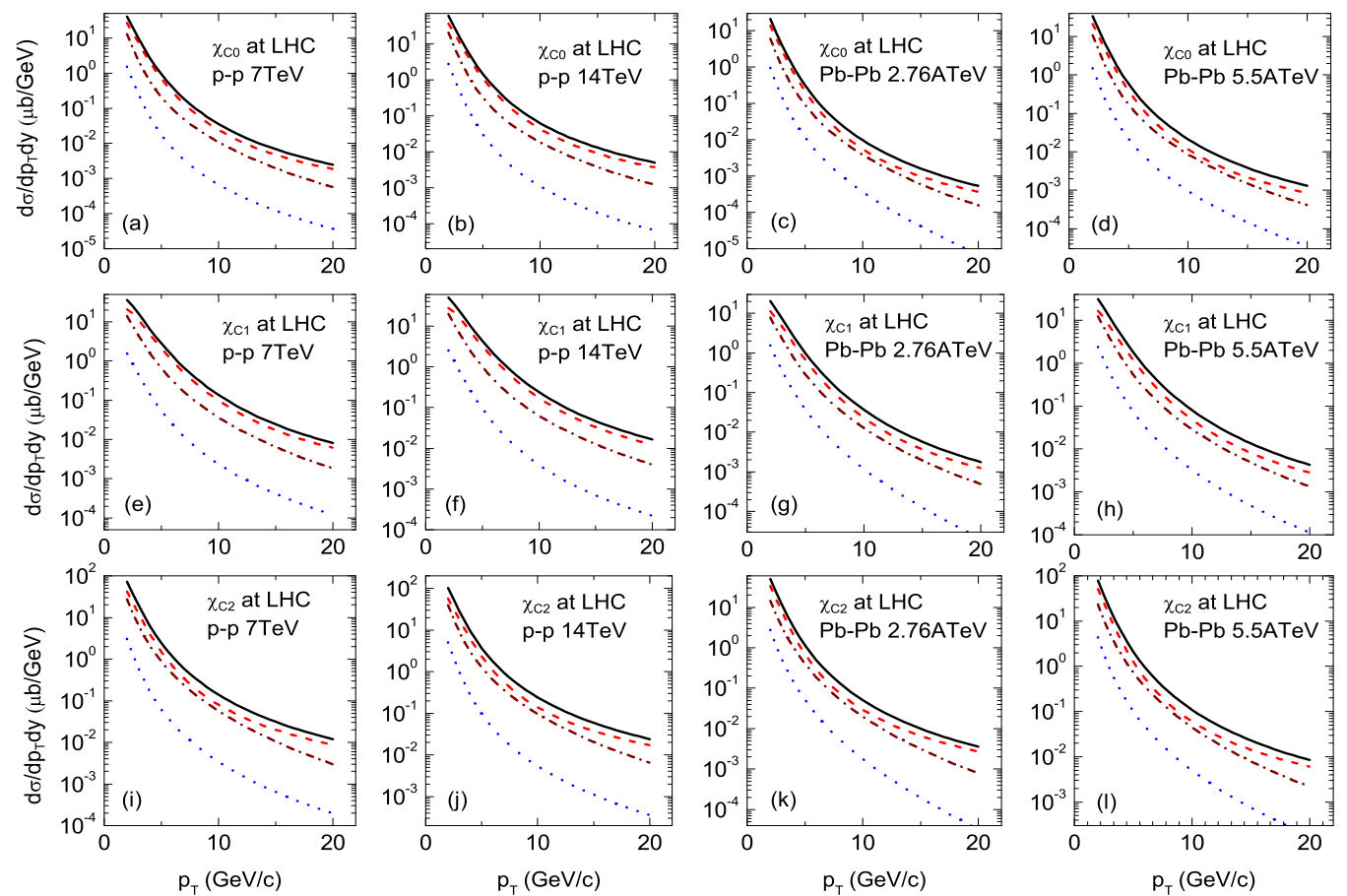

FIG. 2. The same as Fig.1 1 but for $\chi_{c J}$ production in p-p collisions $(\sqrt{s}=7.0 T e V$ and $\sqrt{s}=14.0 T e V)$ and $\mathrm{Pb}-\mathrm{Pb}$ collisions $(\sqrt{s}=2.76 \mathrm{TeV}$ and $\sqrt{s}=5.5 \mathrm{TeV})$ at LHC.

$$
\begin{aligned}
\left|\eta_{b}\right\rangle= & \left\langle\mathcal{O}^{\eta_{b}}\left[{ }^{1} S_{0}^{[1]}\right]\right\rangle\left|Q \bar{Q}\left[{ }^{1} S_{0}^{[1]}\right]\right\rangle \\
& +\left\langle\mathcal{O}^{\eta_{b}}\left[{ }^{1} S_{0}^{[8]}\right]\right\rangle\left|Q \bar{Q}\left[{ }^{1} S_{0}^{[8]}\right] g\right\rangle \\
& +\left\langle\mathcal{O}^{\eta_{b}}\left[{ }^{3} S_{1}^{[8]}\right]\right\rangle\left|Q \bar{Q}\left[{ }^{3} S_{1}^{[8]}\right] g\right\rangle \\
& +\left\langle\mathcal{O}^{\eta_{b}}\left[{ }^{1} P_{1}^{[8]}\right]\right\rangle\left|Q \bar{Q}\left[{ }^{1} P_{1}^{[8]}\right] g\right\rangle \\
& +\cdots \cdots \\
\left|h_{b}\right\rangle= & \left\langle\mathcal{O}^{h_{b}}\left[{ }^{1} P_{1}^{[1]}\right]\right\rangle\left|Q \bar{Q}\left[{ }^{1} P_{1}^{[1]}\right] g\right\rangle \\
& +\left\langle\mathcal{O}^{h_{b}}\left[{ }^{1} S_{0}^{[8]}\right]\right\rangle\left|Q \bar{Q}\left[{ }^{1} S_{0}^{[8]}\right] g\right\rangle \\
& +\cdots \cdots,
\end{aligned}
$$

$$
\begin{aligned}
\left\langle\mathcal{O}^{\Upsilon(2 S)}\left[{ }^{3} S_{1}^{[1]}\right]\right\rangle & =4.5 \mathrm{GeV}^{3}, \\
\left\langle\mathcal{O}^{\Upsilon(2 S)}\left[{ }^{1} S_{0}^{[8]}\right]\right\rangle & =(-0.0067 \pm 0.0084) \mathrm{GeV}^{3}, \\
\left\langle\mathcal{O}^{\Upsilon(2 S)}\left[{ }^{3} S_{1}^{[8]}\right]\right\rangle & =(0.0224 \pm 0.0200) \mathrm{GeV}^{3}, \\
\left\langle\mathcal{O}^{\Upsilon(2 S)}\left[{ }^{3} P_{0}^{[8]}\right]\right\rangle & =5 \times(-0.0067 \pm 0.0084) m_{b}^{2} \cdot \mathrm{GeV}^{3},
\end{aligned}
$$

where $\left\langle\mathcal{O}^{H}\left[{ }^{2 S+1} L_{J}^{[1,8]}\right]\right\rangle$ is the long-distance matrix element for the bottomonium [18, 19, 101-103], and the longdistance matrix elements for the $\Upsilon(n S)$ meson are given by

$$
\begin{aligned}
& \left\langle\mathcal{O}^{\Upsilon(1 S)}\left[{ }^{3} S_{1}^{[1]}\right]\right\rangle=10.9 \mathrm{GeV}^{3}, \\
& \left\langle\mathcal{O}^{\Upsilon(1 S)}\left[{ }^{1} S_{0}^{[8]}\right]\right\rangle=(0.0121 \pm 0.0400) \mathrm{GeV}^{3}, \\
& \left\langle\mathcal{O}^{\Upsilon(1 S)}\left[{ }^{3} S_{1}^{[8]}\right]\right\rangle=(0.0477 \pm 0.0334) \mathrm{GeV}^{3}, \\
& \left\langle\mathcal{O}^{\Upsilon(1 S)}\left[{ }^{3} P_{0}^{[8]}\right]\right\rangle=5 \times(0.0121 \pm 0.0400) \mathrm{m}_{b}^{2} \cdot \mathrm{GeV}^{3},
\end{aligned}
$$

$$
\begin{aligned}
& \left\langle\mathcal{O}^{\Upsilon(3 S)}\left[{ }^{3} S_{1}^{[1]}\right]\right\rangle=4.3 \mathrm{GeV}^{3}, \\
& \left\langle\mathcal{O}^{\Upsilon(3 S)}\left[{ }^{1} S_{0}^{[8]}\right]\right\rangle=(0.0002 \pm 0.0062) \mathrm{GeV}^{3}, \\
& \left\langle\mathcal{O}^{\Upsilon(3 S)}\left[{ }^{3} S_{1}^{[8]}\right]\right\rangle=(0.0513 \pm 0.0085) \mathrm{GeV}^{3}, \\
& \left\langle\mathcal{O}^{\Upsilon(3 S)}\left[{ }^{3} P_{0}^{[8]}\right]\right\rangle=5 \times(0.0002 \pm 0.0062) \mathrm{m}_{b}^{2} \cdot \mathrm{GeV}^{3},
\end{aligned}
$$



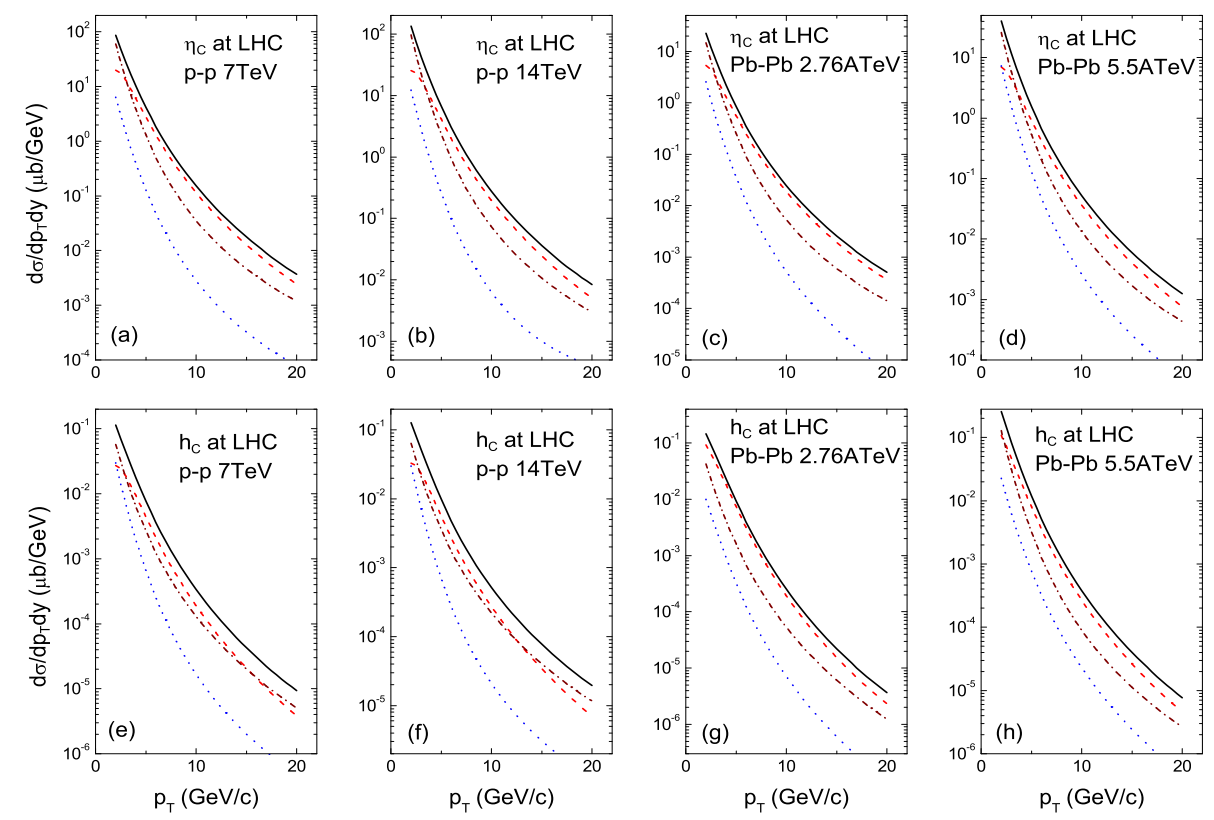

FIG. 3. The same as Fig. 1 but for $\eta_{c}$ and $h_{c}$ production in p-p collisions $(\sqrt{s}=7.0 T e V$ and $\sqrt{s}=14.0 T e V)$ and $\mathrm{Pb}-\mathrm{Pb}$ collisions $(\sqrt{s}=2.76 \mathrm{TeV}$ and $\sqrt{s}=5.5 \mathrm{TeV})$ at LHC.

for $\chi_{b J}$ meson,

$$
\begin{aligned}
& \left\langle\mathcal{O}^{\chi_{b 0}(1 P)}\left[{ }^{3} P_{0}^{[1]}\right]\right\rangle=0.1 m_{b}^{2} \cdot G e V^{3}, \\
& \left\langle\mathcal{O}^{\chi_{b 0}(1 P)}\left[{ }^{3} S_{1}^{[8]}\right]\right\rangle=0.1008 \mathrm{GeV}^{3}, \\
& \left\langle\mathcal{O}^{\chi_{b 0}(2 P)}\left[{ }^{3} P_{0}^{[1]}\right]\right\rangle=0.036 m_{b}^{2} \cdot \mathrm{GeV}^{3}, \\
& \left\langle\mathcal{O}^{\chi_{b 0}(2 P)}\left[{ }^{3} S_{1}^{[8]}\right]\right\rangle=0.0324 \mathrm{GeV}^{3}, \\
& \left\langle\mathcal{O}^{\chi_{b 1}(1 P)}\left[{ }^{3} P_{1}^{[1]}\right]\right\rangle=6.1 \mathrm{GeV}^{5} \\
& \left\langle\mathcal{O}^{\chi_{b 1}(1 P)}\left[{ }^{3} S_{1}^{[8]}\right]\right\rangle=0.43 \mathrm{GeV}^{3} \\
& \left\langle\mathcal{O}^{\chi_{b 1}(2 P)}\left[{ }^{3} P_{1}^{[1]}\right]\right\rangle=7.1 m_{b}^{2} G e V^{3}, \\
& \left\langle\mathcal{O}^{\chi_{b 1}(2 P)}\left[{ }^{3} S_{1}^{[8]}\right]\right\rangle=0.52 \mathrm{GeV}^{3}, \\
& \left\langle\mathcal{O}^{\chi_{b 1}(3 P)}\left[{ }^{3} P_{1}^{[1]}\right]\right\rangle=7.7 m_{b}^{2} \mathrm{GeV}^{3},
\end{aligned}
$$

for $\eta_{b}$ meson,

$$
\begin{aligned}
\left\langle\mathcal{O}^{\eta_{b}(n S)}\left[{ }^{1} S_{0}^{[1]}\right]\right\rangle & =\frac{1}{3} \times\left\langle\mathcal{O}^{\Upsilon(n S)}\left[{ }^{3} S_{1}^{[1]}\right]\right\rangle, \\
\left\langle\mathcal{O}^{\eta_{b}(n S)}\left[{ }^{1} S_{0}^{[8]}\right]\right\rangle & =\frac{1}{3} \times\left\langle\mathcal{O}^{\Upsilon(n S)}\left[{ }^{3} S_{1}^{[8]}\right]\right\rangle, \\
\left\langle\mathcal{O}^{\eta_{b}(n S)}\left[{ }^{3} S_{1}^{[8]}\right]\right\rangle & =\left\langle\mathcal{O}^{\Upsilon(n S)}\left[{ }^{1} S_{0}^{[8]}\right]\right\rangle, \\
\left\langle\mathcal{O}^{\eta_{b}(n S)}\left[{ }^{1} P_{1}^{[8]}\right]\right\rangle & =3 \times\left\langle\mathcal{O}^{\Upsilon(n S)}\left[{ }^{3} P_{0}^{[8]}\right]\right\rangle,
\end{aligned}
$$

and for $h_{b}$ meson,

$$
\begin{aligned}
& \left\langle\mathcal{O}^{h_{b}(n P)}\left[{ }^{1} P_{1}^{[1]}\right]\right\rangle=3 \times\left\langle\mathcal{O}^{\chi_{b 0}(n P)}\left[{ }^{3} P_{0}^{[1]}\right]\right\rangle, \\
& \left\langle\mathcal{O}^{h_{b}(n P)}\left[{ }^{1} S_{0}^{[8]}\right]\right\rangle=3 \times\left\langle\mathcal{O}^{\chi_{b 0}(n P)}\left[{ }^{3} S_{1}^{[8]}\right]\right\rangle,
\end{aligned}
$$

here $m_{b}$ is the bottom quark mass.

In the non-relativistic Quantum Chromodynamics (NRQCD) factorization approach, the hadroproduction cross section for the heavy quarkonium produced by the initial parton hard scattering processes (LO) can be expressed as

$$
\begin{aligned}
& \frac{d \sigma_{A B \rightarrow H X}^{L O}}{d p_{T}^{2} d y}=\sum_{n} \frac{d \sigma_{A B \rightarrow Q \bar{Q}_{[1,8]}(n)+X}^{L O}}{d p_{T}^{2} d y}\left\langle\mathcal{O}_{[1,8]}^{H}[n]\right\rangle \\
& =\sum_{n} \int d x_{a} f_{A}\left(x_{a}, Q^{2}\right) f_{B}\left(x_{b}, Q^{2}\right) \frac{x_{a} x_{b}}{x_{a}-x_{1}} \\
& \times \frac{d \hat{\sigma}}{d \hat{t}}\left(a+b \rightarrow Q \bar{Q}_{[1,8]}[n]+X\right)\left\langle\mathcal{O}_{[1,8]}^{H}[n]\right\rangle,
\end{aligned}
$$

where $x_{a}$ and $x_{b}=\left(x_{a} x_{2}-\tau\right) /\left(x_{a}-x_{1}\right)$ are the momentum fractions of the partons. The variables are $x_{1}=\frac{1}{2}\left(x_{T}^{2}+4 \tau\right)^{1 / 2} \exp (y), x_{2}=\frac{1}{2}\left(x_{T}^{2}+4 \tau\right)^{1 / 2} \exp (-y)$, $x_{T}=2 p_{T} / \sqrt{s}, \tau=M^{2} / s$, and $M$ is the mass of the heavy quarkonium.

The parton distribution function $f_{A}\left(x, Q^{2}\right)$ of the nucleon is given by 104 107]

$$
f_{A}\left(x, Q^{2}\right)=R_{A}\left(x, Q^{2}\right)\left[\frac{Z}{A} p\left(x, Q^{2}\right)+\frac{N}{A} n\left(x, Q^{2}\right)\right],
$$



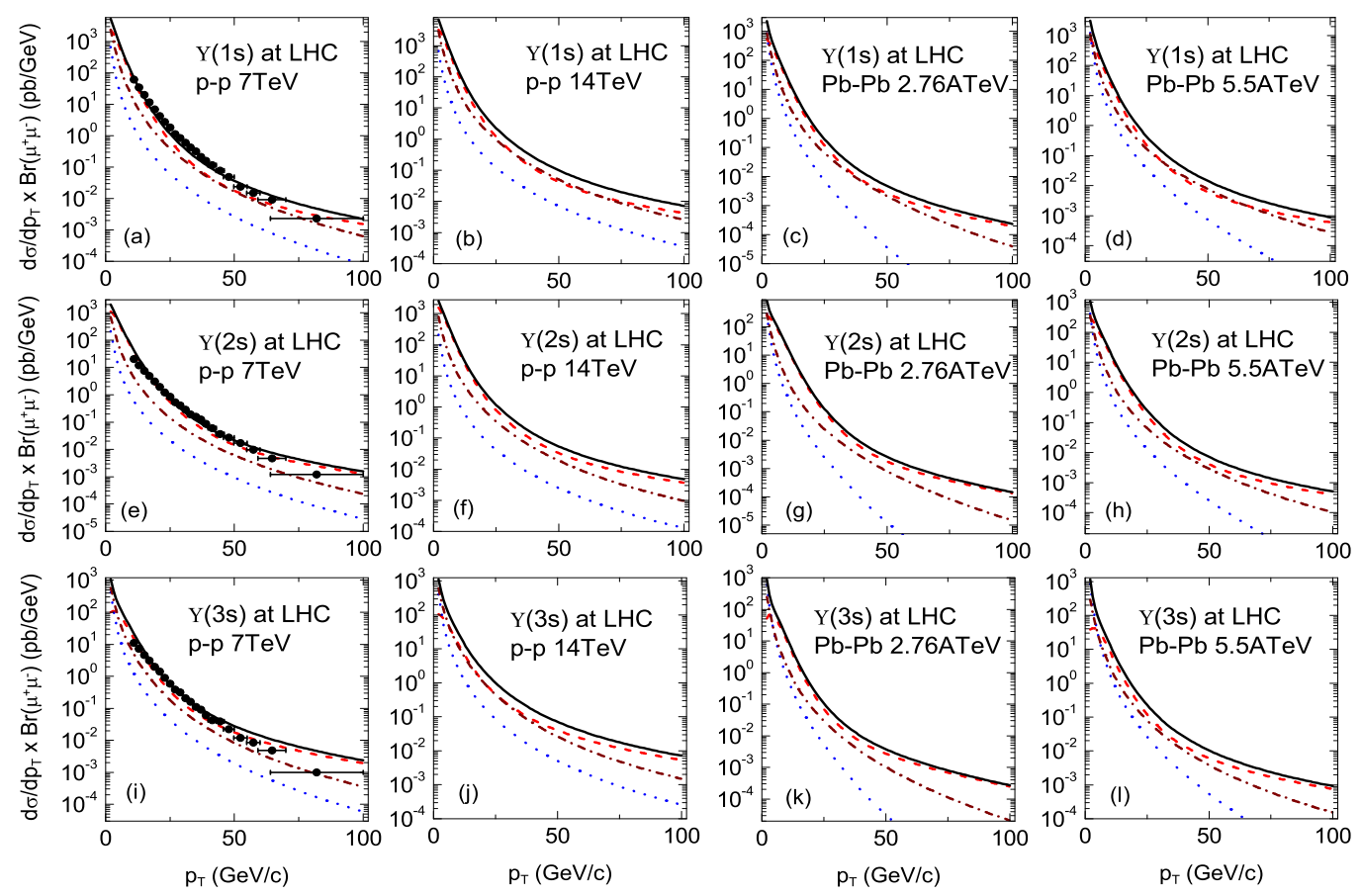

FIG. 4. The invariant cross section of large- $p_{T} \Upsilon(n S)$ production for p-p collisions $(\sqrt{s}=7.0 T e V$ and $\sqrt{s}=14.0 T e V)$ and $\mathrm{Pb}-\mathrm{Pb}$ collisions $(\sqrt{s}=2.76 \mathrm{TeV}$ and $\sqrt{s}=5.5 \mathrm{TeV})$ at LHC. The dashed line (red line) for the initial parton hard scattering processes, the dotted line (blue line) for the semielastic photoproduction processes, the dashed-dotted line (wine line) for the inelastic photoproduction processes. The branching fractions are $B_{r}\left(\Upsilon(1 S) \rightarrow \mu^{+} \mu^{-}\right)=2.48 \%, B_{r}\left(\Upsilon(2 S) \rightarrow \mu^{+} \mu^{-}\right)=1.93 \%$, and $B_{r}\left(\Upsilon(3 S) \rightarrow \mu^{+} \mu^{-}\right)=2.18 \%$. The $\Upsilon(n S)$ data points are from the CMS Collaboration [9].

where $R_{A}\left(x, Q^{2}\right)$ is the nuclear modification factor [108], $Z$ is the proton number, $N$ is the neutron number, and $A$ is the nucleon number; $p\left(x, Q^{2}\right)$ and $n\left(x, Q^{2}\right)$ are the parton distribution functions of protons and neutrons, respectively.

The differential cross section of the initiated partonic subprocesses was calculated in Ref.[77] and can be written as

$$
\begin{aligned}
& \frac{d \hat{\sigma}}{d \hat{t}}(a+b \rightarrow H+X) \\
& =\frac{d \hat{\sigma}}{d \hat{t}}\left(a+b \rightarrow Q \bar{Q}_{[1,8]}[n]+X\right)\left\langle\mathcal{O}_{[1,8]}^{H}[n]\right\rangle \\
& =\frac{1}{16 \pi \hat{s}^{2}}\left|M\left[^{2 S+1} L_{J}^{[1,8]}\right]\right|^{2} \frac{\left\langle 0\left|\mathcal{O}^{H}\left[{ }^{2 S+1} L_{J}^{[1,8]}\right]\right| 0\right\rangle}{m_{Q}(2 J+1)},
\end{aligned}
$$

where $m_{Q}$ is the mass of the heavy flavor quark.

The fragmentation cross section for the heavy quarkonium produced by the initial parton hard scattering pro- cesses (LO-fra.) can be expressed as

$$
\begin{aligned}
& \frac{d \sigma_{A B \rightarrow H X}^{L O-f r a .}}{d p_{T}^{2} d y}=\sum_{n} \frac{d \sigma_{A B \rightarrow Q \bar{Q}_{[1,8]}(n)+X}^{L O-f r a}}{d p_{T}^{2} d y}\left\langle\mathcal{O}_{[1,8]}^{H}[n]\right\rangle \\
& =\sum_{n} \int d x_{a} d x_{b} f_{A}\left(x_{a}, Q^{2}\right) f_{B}\left(x_{b}, Q^{2}\right) \frac{x_{a} x_{b}}{z_{c}\left(x_{a} x_{b}-\tau\right)} \\
& \times \frac{d \hat{\sigma}}{d \hat{t}}(a b \rightarrow c d) D_{c \rightarrow Q \bar{Q}_{[1,8]}[n]}\left(z_{c}, Q^{2}\right)\left\langle\mathcal{O}_{[1,8]}^{H}[n]\right\rangle,
\end{aligned}
$$

where $z_{c}$ is the momentum fraction of the final heavy quarkonium, $\frac{d \hat{\sigma}}{d t}(a b \rightarrow c d)$ is the differential cross section for the subprocess [109], and the fragmentation function $D_{c \rightarrow Q \bar{Q}_{[1,8]}[n]}\left(z_{c}, Q^{2}\right)$ was calculated in Ref. [66 -70$]$.

The large- $p_{T}$ heavy quarkonium produced by semielastic photoproduction processes can be divided into the semielastic direct photoproduction processes and semielastic resolved photoproduction processes in ultrarelativistic heavy ion collisions.

In the semielastic direct photoproduction processes, incident nucleus can emit a photon, then the high-energy photon interacts with parton of another incident nucleus by the interaction of quark-photon Compton scattering and gluon-photon fusion. The differential cross section of heavy quarkonium produced by the semielastic direct 

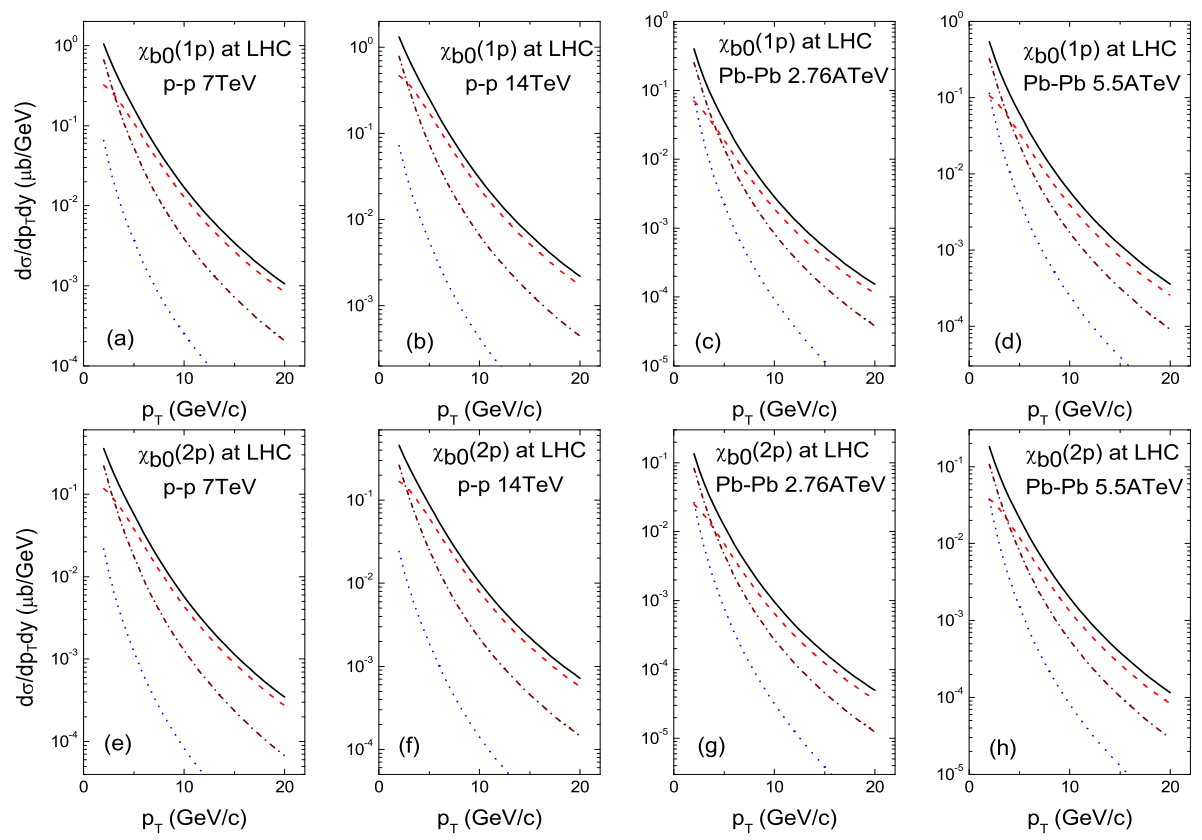

FIG. 5. The same as Fig. 14 but for $\chi_{b 0}$ production in p-p collisions $(\sqrt{s}=7.0 T e V$ and $\sqrt{s}=14.0 T e V)$ and $\mathrm{Pb}-\mathrm{Pb}$ collisions $(\sqrt{s}=2.76 \mathrm{TeV}$ and $\sqrt{s}=5.5 \mathrm{TeV})$ at LHC.

photoproduction processes (semi.dir. and semi.dir.-fra.) can be written as

$$
\begin{aligned}
& \frac{d \sigma_{A B \rightarrow H X}^{\text {semi.dir. }}}{d p_{T}^{2} d y}=\sum_{n} \frac{d \sigma_{A B \rightarrow Q \bar{Q}_{[1,8]}(n)+X}^{\text {semi.dir. }}}{d p_{T}^{2} d y}\left\langle\mathcal{O}_{[1,8]}^{H}[n]\right\rangle \\
= & \sum_{n} \int d x_{a} f_{\gamma / N}\left(x_{a}\right) f_{B}\left(x_{b}, Q^{2}\right) \frac{x_{a} x_{b}}{x_{a}-x_{1}} \\
& \times \frac{d \hat{\sigma}}{d \hat{t}}\left(\gamma+b \rightarrow Q \bar{Q}_{[1,8]}[n]+X\right)\left\langle\mathcal{O}_{[1,8]}^{H}[n]\right\rangle, \\
& \frac{d \sigma_{A B \rightarrow H X}^{\text {semi.dir. }} \text { fra. }}{d p_{T}^{2} d y}=\sum_{n} \frac{d \sigma_{A B \rightarrow Q}^{\text {semi.dir.- fra. }}}{d p_{T}^{2} d y}(n)+X \\
= & \sum_{n} \int d x_{a} d x_{b} f_{\gamma / N}\left(x_{a}\right) f_{B}\left(x_{b}, Q^{2}\right) \frac{x_{a} x_{b}}{z_{c}\left(x_{a} x_{b}-\tau\right)} \\
\times & \frac{d \hat{\sigma}}{d \hat{t}}(\gamma b \rightarrow c d) D_{c \rightarrow Q \bar{Q}_{[1,8]}[n]}\left(z_{c}, Q^{2}\right)\left\langle\mathcal{O}_{[1,8]}^{H}[n]\right\rangle,
\end{aligned}
$$

Here the differential cross section of the subprocesses was calculated in Ref.[77].

The equivalent photon spectrum for nucleus can be obtained from the semiclassical description of high-energy electromagnetic collisions. A relativistic nucleus with $Z$ times the electric charge moving with a relativistic factor $\gamma \gg 1$ with respect to some observers develop an equally strong magnetic field component so it resembles a beam of real photons, where the number of photons can be expressed as [110, 111]

$$
f_{\gamma / N}(\omega)=\frac{2 Z^{2} \alpha}{\pi \omega} \ln \left(\frac{\gamma}{\omega R}\right),
$$

where is the photon momentum, and $R=b_{\min }$ is the radius of the nucleus $\left(b_{\min }\right.$ is the cutoff of impact parameter). In the logarithmic approximation, the results obtained from the purely classical treatment or by including the form factor are related to each other through the rescaling of the relativistic factor.

For p-p collisions, the equivalent photon spectrum function for the proton can be obtained from the Weizsäcker-Williams approximation [112 114],

$$
\begin{aligned}
f_{\gamma / p}(x)= & \frac{\alpha}{2 \pi} \frac{1+(1-x)^{2}}{x} \\
& \times\left[\ln A_{p}-\frac{11}{6}+\frac{3}{A_{p}}-\frac{3}{2 A_{p}^{2}}+\frac{1}{3 A_{p}^{3}}\right],
\end{aligned}
$$

where $x$ is the momentum fraction of the photon, $A_{p}=$ $1+0.71 \mathrm{GeV}^{2} / Q_{\min }^{2}$ with

$$
\begin{aligned}
Q_{\text {min }}^{2}= & -2 m_{p}^{2}+\frac{1}{2 s}\left\{\left(s+m_{p}^{2}\right)\left(s-x s+m_{p}^{2}\right)\right. \\
& \left.-\left(s-m_{p}^{2}\right) \sqrt{\left(s-x s+m_{p}^{2}\right)^{2}-4 m_{p}^{2} x s}\right\},
\end{aligned}
$$

here $m_{p}$ is the the mass of the proton, and at high energies $Q_{m i n}^{2}$ is given to a very good approximation by 

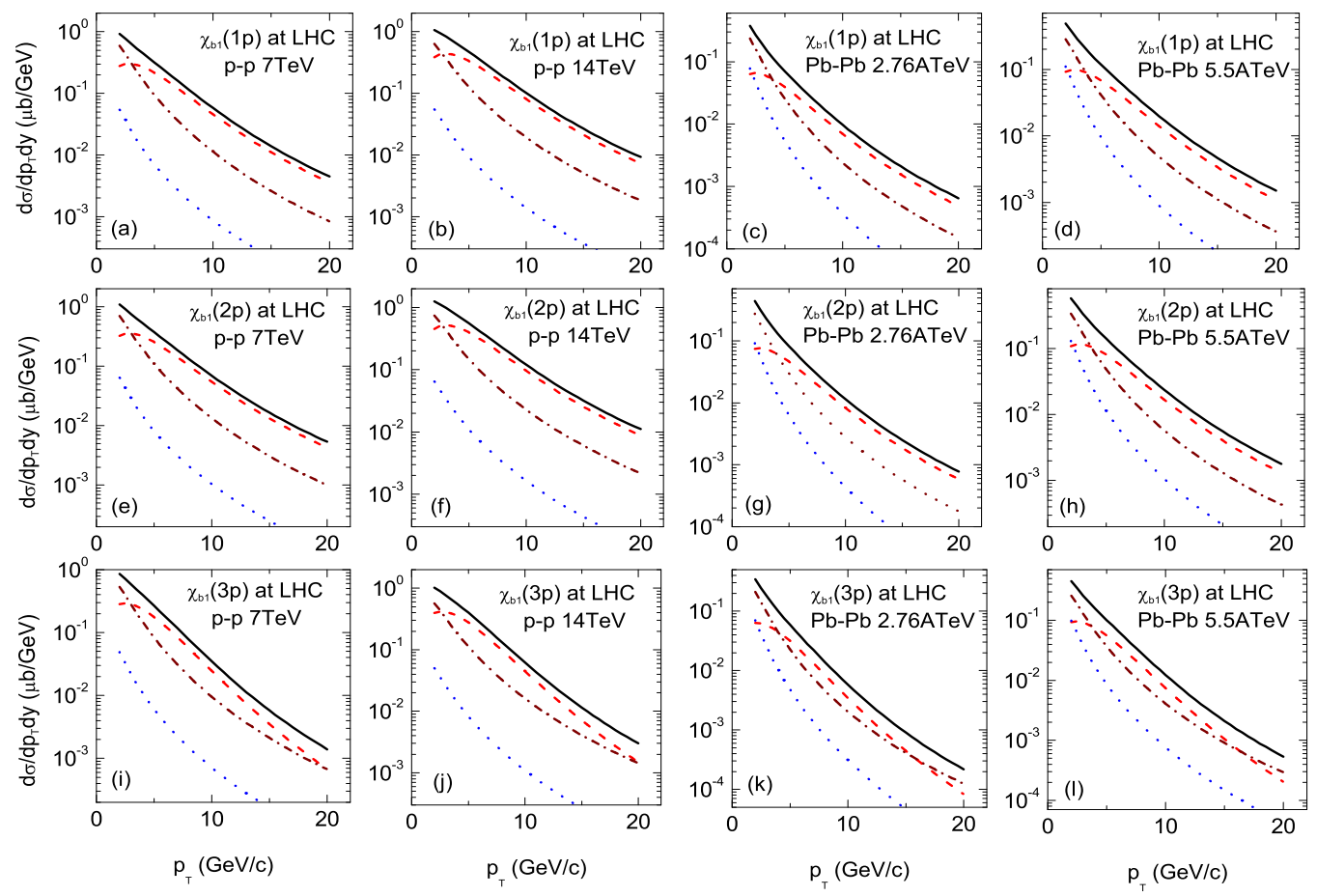

FIG. 6. The same as Fig. \ but for $\chi_{b 1}$ production in p-p collisions $(\sqrt{s}=7.0 T e V$ and $\sqrt{s}=14.0 T e V)$ and $\mathrm{Pb}-\mathrm{Pb}$ collisions $(\sqrt{s}=2.76 \mathrm{TeV}$ and $\sqrt{s}=5.5 \mathrm{TeV})$ at LHC.

$m_{p}^{2} x^{2} /(1-x)$. Propagated uncertainties to the final cross sections are on the order of $10 \%$ for p-p collisions and $20 \%$ for $\mathrm{Pb}-\mathrm{Pb}$ collisions since covering different form-factor parametrizations and the convolution of the nuclear photon fluxes [115].

In the semielastic resolved photoproduction processes, the parton from the hadron-like photon emitted by incident nucleus can interact with the parton of another incident nucleus via the interactions of quark-antiquark annihilation, quark-gluon Compton scattering, and gluongluon fusion. The invariant cross section for large- $p_{T}$ heavy quarkonium produced by the semielastic resolved photoproduction processes (semi.res. and semi.res.-fra.) in the hadronic collisions can be written as

$$
\begin{aligned}
& \frac{d \sigma_{A B \rightarrow H X}^{\text {semi.res. }}}{d p_{T}^{2} d y}=\sum_{n} \frac{d \sigma_{A B \rightarrow Q Q_{[1,8]}^{\text {semi.res. }}}^{\text {sem }}(n)+X}{d p_{T}^{2} d y}\left\langle\mathcal{O}_{[1,8]}^{H}[n]\right\rangle \\
& =\sum_{n} \int d x_{a} d x_{b} f_{\gamma / N}\left(x_{a}\right) f_{\gamma}\left(z_{a}, Q^{2}\right) f_{B}\left(x_{b}, Q^{2}\right) \\
& \times \frac{x_{a} x_{b} z_{a}}{x_{a} x_{b}-x_{a} x_{2}} \frac{d \hat{\sigma}}{d \hat{t}}\left(a^{\prime} b \rightarrow Q \bar{Q}_{[1,8]}[n]+X\right)\left\langle\mathcal{O}_{[1,8]}^{H}[n]\right\rangle,
\end{aligned}
$$

$$
\begin{aligned}
& \frac{d \sigma_{A B \rightarrow H X}^{\text {semi.res.-fra. }}}{d p_{T}^{2} d y}=\sum_{n} \frac{d \sigma_{A B \rightarrow Q \bar{Q}_{[1,8]}(n)+X}^{\text {semi.res. } f r a .}}{d p_{T}^{2} d y}\left\langle\mathcal{O}_{[1,8]}^{H}[n]\right\rangle \\
& =\sum_{n} \int d x_{a} d x_{b} d z_{a} f_{\gamma / N}\left(x_{a}\right) f_{\gamma}\left(z_{a}, Q^{2}\right) f_{B}\left(x_{b}, Q^{2}\right) \\
& \times \frac{x_{a} x_{b} z_{a}}{z_{c}\left(x_{a} x_{b} z_{a}-\tau\right)} \frac{d \hat{\sigma}}{d \hat{t}}\left(a^{\prime} b \rightarrow c d\right) D_{c \rightarrow Q \bar{Q}_{[1,8]}[n]}\left(z_{c}, Q^{2}\right)\left\langle\mathcal{O}_{[1,8]}^{H}[n]\right\rangle,
\end{aligned}
$$

where $f_{\gamma}\left(z_{a}, Q^{2}\right)$ is the parton distribution function of the hadron-like photon [116], the differential cross section of the subprocesses was calculated in Ref.[77].

The large- $p_{T}$ heavy quarkonium produced by inelastic photoproduction processes can be divided into the inelastic direct photoproduction processes and inelastic resolved photoproduction processes in ultrarelativistic heavy ion collisions.

In the inelastic direct photoproduction processes, the charged parton of the incident nucleus can emit a photon, then the high energy photon interacts with parton of another incident nucleus by the interaction of quark-photon Compton scattering and gluon-photon fusion. The differential cross section of heavy quarkonium produced by the inelastic direct photoproduction processes (inel.dir. and inel.dir.-fra.) in the hadronic collisions can be expressed 

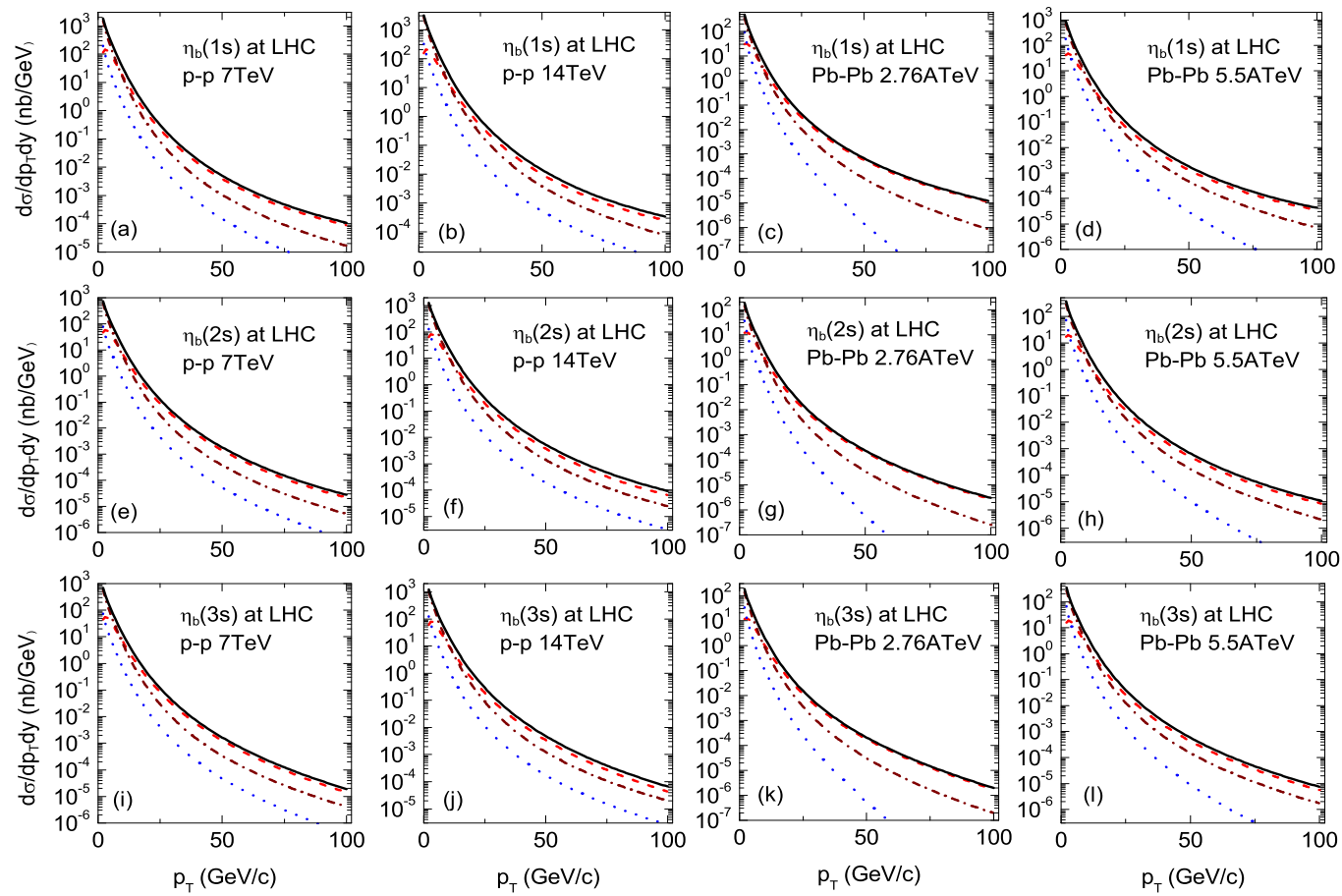

FIG. 7. The same as Fig. (4 but for $\eta_{b}$ production in p-p collisions $(\sqrt{s}=7.0 T e V$ and $\sqrt{s}=14.0 T e V)$ and $\mathrm{Pb}-\mathrm{Pb}$ collisions $(\sqrt{s}=2.76 \mathrm{TeV}$ and $\sqrt{s}=5.5 \mathrm{TeV})$ at LHC.

as

$$
\begin{aligned}
& \frac{d \sigma_{A B \rightarrow H X}^{\text {inel.dir. }}}{d p_{T}^{2} d y}=\sum_{n} \frac{d \sigma_{A B \rightarrow Q}^{\text {inel.dir. }} \bar{Q}_{[1,8]}(n)+X}{d p_{T}^{2} d y}\left\langle\mathcal{O}_{[1,8]}^{H}[n]\right\rangle \\
& =\sum_{n} \int d x_{a} d x_{b} f_{A}\left(x_{a}, Q^{2}\right) f_{\gamma / q}\left(z_{a}\right) f_{B}\left(x_{b}, Q^{2}\right) \\
& \times \frac{x_{a} x_{b} z_{a}}{x_{a} x_{b}-x_{a} x_{2}} \frac{d \hat{\sigma}}{d \hat{t}}\left(\gamma b \rightarrow Q \bar{Q}_{[1,8]}[n]+X\right)\left\langle\mathcal{O}_{[1,8]}^{H}[n]\right\rangle,
\end{aligned}
$$

$\frac{d \sigma_{A B \rightarrow H X}^{\text {inel.dir. } f r a .}}{d p_{T}^{2} d y}=\sum_{n} \frac{d \sigma_{A B \rightarrow Q \bar{Q}_{[1,8]}^{\text {inel.dir. }}(n)+X}}{d p_{T}^{2} d y}\left\langle\mathcal{O}_{[1,8]}^{H}[n]\right\rangle$

$=\sum_{n} \int d x_{a} d x_{b} d z_{a} f_{A}\left(x_{a}, Q^{2}\right) f_{\gamma / q}\left(z_{a}\right) f_{B}\left(x_{b}, Q^{2}\right)$

$\times \frac{x_{a} x_{b} z_{a}}{z_{c}\left(x_{a} x_{b} z_{a}-\tau\right)} \frac{d \hat{\sigma}}{d \hat{t}}(\gamma b \rightarrow c d) D_{c \rightarrow Q \bar{Q}_{[1,8]}[n]}\left(z_{c}, Q^{2}\right)\left\langle\mathcal{O}_{[1,8]}^{H}[n]\right\rangle$,

here the equivalent photon spectrum function of the charged parton is given by [117, 118]

$$
\begin{aligned}
f_{\gamma / q}(x)= & \frac{\alpha}{2 \pi} e_{f}^{2}\left\{\frac{1+(1-x)^{2}}{x}\left[\ln \left(\frac{E}{m}\right)-\frac{1}{2}\right]\right. \\
& \left.+\frac{x}{2}\left[\ln \left(\frac{2}{x}-2\right)+1\right]+\frac{(2-x)^{2}}{2 x} \ln \left(\frac{2-2 x}{2-x}\right)\right\},
\end{aligned}
$$

where $x$ is the the photon momentum fraction. The variables $e_{f}, E$, and $m$ are the charge, energy, and mass of the parton, respectively.

In the inelastic resolved photoproduction processes, the parton from the hadron-like photon emitted by the charged parton of incident nucleus can interact with the parton of another incident nucleus via the interactions of quark-antiquark annihilation, quark-gluon Compton scattering, and gluon-gluon fusion. The invariant cross section for large- $p_{T}$ heavy quarkonium produced by the inelastic resolved photoproduction processes (inel.res. and inel.res.-fra.) in the hadronic collisions can be written as

$$
\begin{aligned}
& \frac{d \sigma_{A B \rightarrow H X}^{\text {inel.res. }}}{d p_{T}^{2} d y}=\sum_{n} \frac{d \sigma_{A B \rightarrow Q \bar{Q}_{[1,8]}^{\text {inel.dir. }}}(n)+X}{d p_{T}^{2} d y}\left\langle\mathcal{O}_{[1,8]}^{H}[n]\right\rangle \\
& =\sum_{n} \int d x_{a} d x_{b} d z_{a} f_{A}\left(x_{a}, Q^{2}\right) f_{\gamma / q}\left(z_{a}\right) f_{\gamma}\left(z_{a}^{\prime}, Q^{2}\right) f_{B}\left(x_{b}, Q^{2}\right) \\
& \times \frac{x_{a} x_{b} z_{a} z_{a}^{\prime}}{x_{a} x_{b} z_{a}-x_{a} z_{a} x_{2}} \frac{d \hat{\sigma}}{d \hat{t}}\left(a^{\prime} b \rightarrow Q \bar{Q}_{[1,8]}[n]+X\right)\left\langle\mathcal{O}_{[1,8]}^{H}[n]\right\rangle,
\end{aligned}
$$



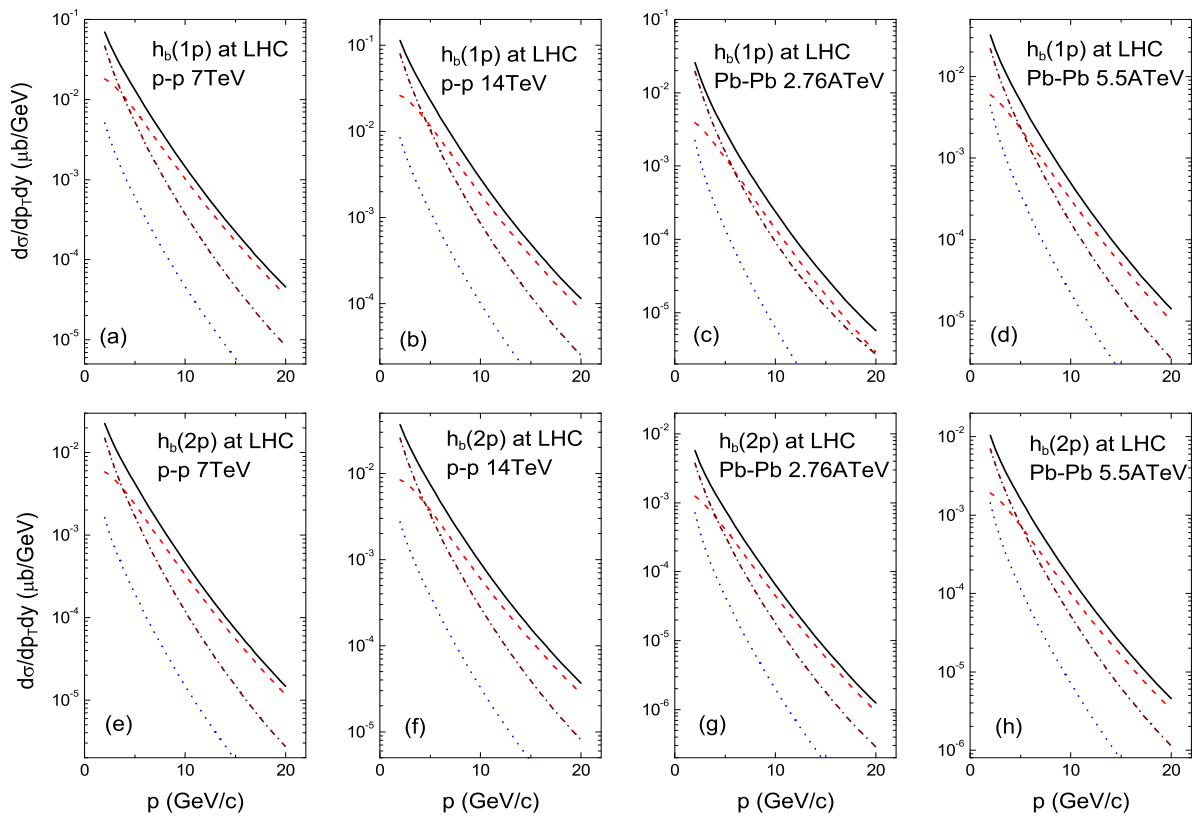

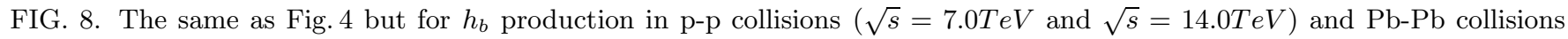
$(\sqrt{s}=2.76 \mathrm{TeV}$ and $\sqrt{s}=5.5 \mathrm{TeV})$ at LHC.

$$
\begin{aligned}
& \frac{d \sigma_{A B \rightarrow H X}^{\text {inel.res.-fra. }}}{d p_{T}^{2} d y}=\sum_{n} \frac{d \sigma_{A B \rightarrow Q \bar{Q}_{[1,8]} \text { inel.res. }}^{\text {fra }}(n)+X}{d p_{T}^{2} d y}\left\langle\mathcal{O}_{[1,8]}^{H}[n]\right\rangle \\
& =\sum_{n} \int d x_{a} d x_{b} d z_{a} d z_{a}^{\prime} f_{A}\left(x_{a}, Q^{2}\right) f_{\gamma / q}\left(z_{a}\right) f_{\gamma}\left(z_{a}^{\prime}, Q^{2}\right) f_{B}\left(x_{b}, Q^{2}\right) \\
& \times \frac{x_{a} x_{b} z_{a} z_{a}^{\prime}}{z_{c}\left(x_{a} x_{b} z_{a} z_{a}^{\prime}-\tau\right)} \frac{d \hat{\sigma}}{d \hat{t}}\left(a^{\prime} b \rightarrow c d\right) D_{c \rightarrow Q \bar{Q}_{[1,8]}[n]}\left(z_{c}, Q^{2}\right)\left\langle\mathcal{O}_{[1,8]}^{H}[n]\right\rangle,
\end{aligned}
$$

where $z_{a}^{\prime}$ is the momentum fraction of the parton from the hadron-like photon.

\section{NUMERICAL RESULTS}

In ultrarelativistic heavy ion collisions, the equivalent photon spectrum for the nucleus obtained from semiclassical description of high energies electromagnetic collisions is $f_{\gamma / N} \propto Z^{2} \ln \gamma$, the relativistic factor $\gamma=$ $E / m_{N}=\sqrt{s} / 2 m_{N} \gg 1$ becomes very large at Large Hadron Collider energies. Indeed, the equivalent photon spectrum function for the proton obtained from Weizsäcker-Williams approximation is $f_{\gamma / p} \propto \ln A \propto$ $\ln \left(s / m_{p}^{2}\right)$, where $m_{p}$ is the proton mass. Since the collision energy $\sqrt{s}$ at Large Hadron Collider is very large, the photon spectrum becomes important. Therefore the contribution of semielastic photoproduction processes is evident at Large Hadron Collider energies. For the inelastic photoproduction processes, the equivalent photon spectrum function of the charged parton is $f_{\gamma / q} \propto$ $\ln \left(E / m_{q}\right)=\ln \left(\sqrt{s} / 2 m_{q}\right)+\ln (x)$, where $m_{q}$ is the charged parton mass. Hence the photon spectrum for the charged parton becomes prominent at Large Hadron Collider energies. Therefore the contribution of photoproduction processes is evident at LHC. Moreover, we also calculate the fragmentation contribution of heavy quarkonium. For inclusive heavy quarkonium production, there is a large contribution from the $g \rightarrow H$ fragmentation, where $H$ is the heavy quarkonium. At large transverse momentum, this is comparable to the direct production processes over almost the whole range of transverse momentum values considered. The numerical results of our calculation for large- $p_{T}$ heavy quarkonium produced by the hard photoproduction processes in relativistic heavy ion collisions are plotted in Figs.1.8.

In Figs.113, we plot the contribution of the charmo$\operatorname{nium}\left(J / \psi, \psi(2 s), \chi_{c J}, \eta_{c}\right.$, and $\left.h_{c}\right)$ produced by the semielastic and inelastic hard photoproduction (and fragmentation) processes for p-p collisions $(\sqrt{s}=7 \mathrm{TeV}$ and $\sqrt{s}=14 T e V)$ and $\mathrm{Pb}-\mathrm{Pb}$ collisions $(\sqrt{s}=2.76 \mathrm{TeV}$ and $\sqrt{s}=5.5 \mathrm{TeV}$ ) in relativistic heavy ion collisions. The charmonium spectra of semielastic and inelastic photoproduction (and fragmentation) processes (the dotted 
line and dashed-dotted line) are compared with the charmonium spectra of the initial parton hard scattering processes (the dashed line) for $\mathrm{p}-\mathrm{p}$ collisions and $\mathrm{Pb}-\mathrm{Pb}$ collisions at the $\mathrm{LHC}$, respectively. In panel (a) and (e) of Figs.1] we compare with the ALICE Collaboration $J / \psi$ meson data[1] and the CMS Collaboration $\psi(2 S)$ meson data[8] in p-p collisions with $\sqrt{s}=7 \mathrm{TeV}$. We find that the contribution of $J / \psi$ and $\psi(2 S)$ meson produced by hard photoproduction and fragmentation processes cannot be negligible for large- $p_{T}$ in $\mathrm{p}-\mathrm{p}$ collisions with $\sqrt{s}=7 T e V$ at LHC [Figs.11(a) and (e)]. We also plot the spectra of bottomonium $\left(\Upsilon(n s), \chi_{b J}, \eta_{b}\right.$, and $h_{b}$ ) produced by the hard photoproduction and fragmentation processes for p-p collisions $(\sqrt{s}=7 \mathrm{TeV}$ and $\sqrt{s}=14 T e V)$ and $\mathrm{Pb}-\mathrm{Pb}$ collisions $(\sqrt{s}=2.76 T e V$ and $\sqrt{s}=5.5 T e V)$ in Figs.4 8 . Compared with the production cross section of the initial parton hard scattering processes (the dashed line), the contribution of the bottomonium produced by semielastic and inelastic hard photoproduction processes (the dotted line and dasheddotted line) is prominent at LHC energies. Furthermore, we compare with the CMS Collaboration $\Upsilon(n S)$ meson data 9] for p-p collisions with $\sqrt{s}=7 \mathrm{TeV}$ in panel (a), (e), and (i) of Figs.4. We find that the contribution of $\Upsilon(n S)$ meson produced by photoproduction processes and fragmentation becomes is evident for $\mathrm{p}$-p collisions with $\sqrt{s}=7 \mathrm{TeV}$ at LHC[Figs.4(a), (e), and (i)].

\section{CONCLUSION}

In the framework of the non-relativistic Quantum Chromodynamics (NRQCD) formalism, we have inves- tigated the production of heavy quarkonium by the semielastic hard photoproduction processes, inelastic hard photoproduction processes and fragmentation processes in $\mathrm{p}-\mathrm{p}$ collisions and $\mathrm{Pb}-\mathrm{Pb}$ collisions in ultrarelativistic heavy ion collisions. At the early stage of relativistic heavy ion collisions, the incident nucleus (the charged parton of the incident nucleus) can emit large$p_{T}$ photons then the high energy photons interact with the partons of another incident nucleon by the quarkphoton Compton scattering and photon-gluon fusion interactions. Furthermore, the partons of the hadron-like photons can interact with the partons of the nucleus by the quarkantiquark annihilation and quark-gluon Compton scattering interactions. Indeed, we also calculate the fragmentation contribution of heavy quarkonium. For inclusive heavy quarkonium production, the contribution of $g \rightarrow H$ fragmentation processes is comparable to the direct production processes over almost the whole range of transverse momentum values considered. The numerical results indicate that the contribution of heavy quarkonium produced by the hard photoproduction processes and fragmentation processes becomes evident in p-p collisions $(\sqrt{s}=7 T e V$ and $\sqrt{s}=14.0 T e V)$ and $\mathrm{Pb}-\mathrm{Pb}$ collisions $(\sqrt{s}=2.76 \mathrm{TeV}$ and $\sqrt{s}=5.5 \mathrm{TeV})$ at Large Hadron Collider (LHC) energies.

\section{ACKNOWLEDGEMENTS}

This work is supported by the National Basic Research Program of China (973 Program) with Grant No. 2014CB845405 and the National Natural Science Foundation of China with Grant No. 11465021 and No. 11065010.
[1] K. Aamodt et al. (ALICE Collaboration), Phys. Lett. B 704, 442 (2011).

[2] B. Abelev et al. (ALICE Collaboration), Phys. Lett. B 718,1273 (2013).

[3] B. Abelev et al. (ALICE Collaboration), Phys. Lett. B 734,314 (2014).

[4] B. Abelev et al. (ALICE Collaboration), Phys. Lett. B 738, 361 (2014).

[5] B. Abelev et al. (ALICE Collaboration), Phys. Lett. B 740, 105 (2015).

[6] C. Silvestre et al. (CMS Collaboration), J. Phys. G: Nucl. Part. Phys. 38, 124033 (2011).

[7] S. Chatrchyan et al. (CMS Collaboration), Phys. Lett. B 727, 101 (2013).

[8] V. Khachatryan et al. (CMS Collaboration), Phys. Rev. Lett. 114, 191802 (2015).

[9] V. Khachatryan et al. (CMS Collaboration), Phys. Lett. B 749, 14 (2015).

[10] G. Aad et al. (ATLAS collaboration), J. High Energy Phys. 07, 154 (2014).

[11] G. Aad et al. (ATLAS collaboration), Phys. Rev. C 92, 034904 (2015).
[12] R. Aaij et al. (LHCb Collaboration), Phys. Lett. B 714, 215 (2012).

[13] R. Aaij et al. (LHCb Collaboration), J. High Energy Phys. 10, 115 (2013).

[14] R. Aaij et al. (LHCb Collaboration), Eur. Phys. J. C 75, 311 (2015).

[15] A. Petrelli, M. Cacciari, M. Greco, F. Maltoni, and M. L. Mangano, Nucl. Phys. B 514, 245 (1998).

[16] E. Braaten and S. Fleming, Phys. Rev. Lett. 74, 3327 (1995).

[17] B. Cano-Coloma and M. A. Sanchis-Lozano, Nucl. Phys. B 508, 753 (1997).

[18] P. Cho and A. K. Leibovich, Phys. Rev. D 53, 150 (1996).

[19] P. Cho and A. K. Leibovich, Phys. Rev. D 53, 6203 (1996).

[20] P. Ko, J. Lee, and H. S. Song, Phys. Rev. D 54, 4312 (1996).

[21] M. Klasen, B. A. Kniehl, L. N. Mihaila, and M. Steinhauser, Phys. Rev. Lett. 89, 032001 (2002).

[22] G. C. Nayak, M. X. Liu, and F. Cooper, Phys. Rev. D 68, 034003 (2003).

[23] H. Fujii, K. Watanabe, Nucl. Phys. A 915, 1 (2013). 
[24] R. M. Godbole, A. Misra, A. Mukherjee, and V. S. Rawoot, Nucl. Phys. B (Proc. Suppl.) 251-252, 56 (2014).

[25] T. Song, Phys. Rev. C 89, 044903 (2014).

[26] J.-W. Qiu, P. Sun, B.-W. Xiao, and F. Yuan, Phys. Rev. D 89, 034007 (2014).

[27] D. E. Kharzeev, E. M. Levin, and K. Tuchin, Nucl. Phys. A 924, 47 (2014).

[28] V. P. Goncalves, B. D. Moreira, and F. S. Navarra, Phys. Lett. B 742, 172 (2015).

[29] L. S. Kisslinger, M. X. Liu, and P. McGaughey, Phys. Rev. C 89, 024914 (2014).

[30] E. G. Ferreiro, Phys. Lett. B 731, 57 (2014).

[31] S. Cho, Phys. Rev. C 91, 054914 (2015).

[32] S. Ganesh and M. Mishra, Nucl. Phys. A 947, 38 (2016).

[33] M. Krämer, Nucl. Phys. B 459, 3 (1996).

[34] M. Krämer, Nucl. Phys. B (Proc. Suppl.) 51C, 195 (1996).

[35] M. Cacciari and M. Krämer, Phys. Rev. Lett. 76, 4128 (1996).

[36] M. Butenschön and B. A. Kniehl, Phys. Rev. Lett. 104, 0720012010.

[37] V. P. Goncalves and M. V. T. Machado, Phys. Rev. Lett. 84, 011902(R) (2011).

[38] V. Rebyakova, M. Strikman, and M. Zhalovc, Phys. Lett. B 710, 647 (2012).

[39] A. Adeluyi and C. A. Bertulani, Phys. Rev. C 85, 044904 (2012).

[40] A. Cisek, W. Schäfer, and A. Szczurek, Phys. Rev. C 86, 014905 (2012).

[41] M. Beneke, M. Krämer, and M. Vänttinen, Phys. Rev. D 57, 4285 (1997).

[42] L.-K. Hao, F. Yuan, and K.-T. Chao, Phys. Rev. Lett. 83, 4490 (1999).

[43] J. Hüfner, Y. P. Ivanov, B. Z. Kopeliovich, and A. V. Tarasov, Phys. Rev. D 62, 094022 (2000).

[44] T. Lappi and H. Mäntysaari, Phys. Rev. C 87, 032201(R) (2013).

[45] G. Sampaio dos Santos and M. V. T. Machado, Phys. Rev. C 89, 025201 (2014).

[46] V. P. Goncalves and W. K. Sauter, Phys. Rev. D 94, 094014 (2015).

[47] V. P. Goncalves and G. G. da Silveira, Phys. Rev. D 91, 054013 (2015).

[48] N. Armesto and A. H. Rezaeian, Phys. Rev. D 90, 054003 (2014).

[49] G. Chen, X.-G. Wu, H.-B. Fu, H.-Y. Han, and Z. Sun, Phys. Rev. D 90, 034004 (2014).

[50] N. Brambilla, A. Pineda, J. Soto, and A. Vairo, Nucl. Phys. B 566, 275 (2000).

[51] B. A. Kniehl, A. A. Penin, V. A. Smirnov, and M. Steinhauser, Nucl. Phys. B 635, 357 (2002).

[52] A. Pineda, Prog. Part. Nucl. Phys. 67, 735 (2012).

[53] J. P. Ma, J. X. Wang, and S. Zhao, Phys. Lett. B 737, 103 (2014).

[54] B. Y. Chen, K. Zhou, and P. F. Zhuang, Phys. Rev. C 86, 034906 (2012).

[55] T. Song, K. C. Han, and C. M. Ko, Nucl. Phys. A 897, 141 (2013).

[56] Y. P. Liu, C. M. Ko, and T. Song, Phys. Lett. B 728, 437 (2014).

[57] L. V. Gribov, E. M. Levin, and M. G. Ryskin, Phys. Rep. 100, 1 (1983).

[58] P. Hagler, R. Kirschner, A. Schafer, L. Szymanowski, and O. V. Teryaev, Phys. Rev. Lett. 86, 1446 (2001).
[59] S. P. Baranov, Phys. Rev. D 66, 114003 (2002).

[60] B. A. Kniehl, D. V. Vasin, and V. A. Saleev, Phys. Rev. D 73, 074022 (2006).

[61] S. P. Baranov, A. V. Lipatov, and N. P. Zotov, Phys. Rev. D 85, 014034 (2012).

[62] E. Braaten and T. C. Yuan, Phys. Rev. Lett. 71, 1673 (1993).

[63] E. Braaten and T. C. Yuan, Phys. Rev. D 50, 3176 (1994).

[64] E. Braaten and T. C. Yuan, Phys. Rev. D 52, 6627 (1995).

[65] P. Cho, M. B. Wise, and S. P. Trivedi, Phys. Rev. D 51, R2039 (1995).

[66] J. P. Ma, Phys. Rev. D 53, 1185 (1996).

[67] E. Braaten and Y.-Q. Chen, Phys. Rev. D 55, 2693 (1997).

[68] G. T. Bodwin, U-Rae Kimb, and J. Lee, J. High Energy Phys. 11, 020 (2012).

[69] Y.-Q. Ma, J.-W. Qiu, and H. Zhang, Phys. Rev. D 89, 094029 (2014).

[70] Y.-Q. Ma, J.-W. Qiu, and H. Zhang, Phys. Rev. D 89, 094030 (2014).

[71] G. T. Bodwin, H. S. Chung, U-Rae Kim, and J. Lee1, Phys. Rev. Lett. 113, 0220012014.

[72] G. T. Bodwin, H. S. Chung, U-Rae Kim, and J. Lee, Phys. Rev. D 92, 074042 (2015).

[73] G. T. Bodwin, H. S. Chung, U-Rae Kim, and J. Lee, Phys. Rev. D 91, 074013 (2015).

[74] P. Artoiseneta and E. Braaten, J. High Energy Phys. 04, 121 (2015).

[75] P. Mathews, P. Poulose, and K. Sridhar, Phys. Lett. B 438, 336 (1998).

[76] K. Hagiwara, E. Koub, and C.-F. Qiao, Phys. Lett. B 570, 39 (2003).

[77] M. Klasen, B. A. Kniehl, L. N. Mihaila, and M. Steinhauser, Phys. Rev. D 68, 034017 (2003).

[78] F. Cooper, M. X. Liu, and G. C. Nayak, Phys. Rev. Lett. 93, 171801 (2004).

[79] P. Artoisenet, J. P. Lansberg, and F. Maltoni, Phys. Lett. B 653, 60 (2007).

[80] M. M. Meijer, J. Smith, and W. L. van Neerven, Phys. Rev. D 77, 034014 (2008).

[81] M. Butenschön and B. A. Kniehl, Phys. Rev. Lett. 106, 022003 (2011).

[82] Z.-B. Kang, J.-W. Qiu, and G. Sterman, Phys. Rev. Lett. 108, 102002 (2012).

[83] B. Gong, L.-P. Wan, J.-X. Wang, and H.-F. Zhang, Phys. Rev. Lett. 112, 032001 (2014).

[84] Z. Sun and X.-G. Wu, Phys. Rev. D 92, 074021 (2015).

[85] Z.-G. He and B. A. Kniehl, Phys. Rev. D 92, 014009 (2015).

[86] S. P. Baranov, Phys. Rev. D 91, 034011 (2015).

[87] Z.-B. Kang, Y.-Q. Ma, J.-W. Qiu, and G. Sterman, Phys. Rev. D 90, 034006 (2014).

[88] Z.-B. Kang, Y.-Q. Ma, J.-W. Qiu, and G. Sterman, Phys. Rev. D 91, 014030 (2015).

[89] Z.-G. He and B. A. Kniehl, Phys. Rev. D 90, 014045 (2014).

[90] M. Butenschoen, Z.-G. He, and B. A. Kniehl, Phys. Rev. Lett. 114, 092004 (2015).

[91] H. Han, Y.-Q. Ma, C. Meng, H.-S. Shao, and K.-T. Chao, Phys. Rev. Lett. 114, 092005 (2015).

[92] H.-F. Zhang, Z. Sun, W.-L. Sang, and R. Li, Phys. Rev. Lett. 114, 092006 (2015). 
[93] Z.-G. He and B. A. Kniehl, Phys. Rev. Lett. 115, 022002 (2015).

[94] J.-X. Wang and H.-F. Zhang, J. Phys. G: Nucl. Part. Phys. 42, 025004 (2015).

[95] Y. Feng, B. Gong, L.-P. Wan, and J.-X. Wang, Chin. Phys. C 39, 123102 (2015).

[96] Y.-D. Li and L.-S. Liu, Phys. Lett. B 377, 177 (1996).

[97] R. Nisius, Phys. Rep. 332, 165 (2000).

[98] M. Krawczyk, A. Zembrzuski, and M. Staszel, Phys. Rep. 345, 265 (2001).

[99] G. T. Bodwin, E. Braaten, and G. P. Lepage, Phys. Rev. D 51, 1125 (1995);

G. T. Bodwin, E. Braaten, G. P. Lepage, Phys. Rev. D 55, 5853 (1997), Erratum.

[100] A. K. Likhoded, A. V. Luchinsky, and S. V. Poslavsky, Phys. Rev. D 86, 074027 (2012).

[101] E. Braaten, S. Fleming and A. K. Leibovich, Phys. Rev. D 63, 094006 (2001).

[102] R. Sharma and I. Vitev, Phys. Rev. C 87, 044905 (2013).

[103] J. L. Domenech and M. A. Sanchis-Lozano, Phys. Lett. B 476, 65 (2000).

[104] M. Glück, E. Reya, and A. Vogt, Z. Phys. C 53, 127 (1992).

[105] G.-M. Yu and Y.-D. Li, Chin. Phys. Lett. 30, 011201 (2013).
[106] G.-M. Yu and Y.-D. Li, Chin. Phys. Lett. 31, 011202 (2014).

[107] G.-M. Yu and Y.-D. Li, Phys. Rev. C 91, 044908 (2015).

[108] X.-N. Wang and M. Gyulassy, Phys. Rev. D 44, 3501 (1991).

[109] J. F. Owens, Rev. Mod. Phys. 59, 465 (1987).

[110] J. D. Jackson, Classical Electrodynamics (Wiley, New York, 1963).

[111] E. Papageorgiu, Phys. Lett. B 250, 155 (1990).

[112] N. Baron and G. Baur, Phys. Rev. C 49, 1127 (1994).

[113] M. Drees, R. M. Godbole, M. Nowakowski, and S. D. Rindani, Phys. Rev. D 50, 2335 (1994).

[114] M. Drees and D. Zeppenfeld, Phys. Rev. D 39, 2536 (1989).

[115] D. d'Enterria and G. G. da Silveira, Phys. Rev. Lett. 111, 080405 (2013).

[116] M. Glück, E. Reya, and I. Schienbein, Phys. Rev. D 60, 054019 (1999).

[117] B. A. Kniehl, Phys. Lett. B 254, 267 (1991).

[118] S. J. Brodsky, T. Kinoshita, and H. Terazawa, Phys. Rev. D 4, 1532 (1971). 